\title{
Positive solution of singular BVPs for a system of dynamic equations on time scales
}

\section{Ariadna Lago, Victoria Otero-Espinar ${ }^{*}$ and Tania Pernas}

\section{*Correspondence:}

mvictoria.otero@usc.es

Departamento de Análise

Matemática, Facultade de

Matemáticas, Universidade de

Santiago de Compostela, Galicia,

Spain

\begin{abstract}
This paper is devoted to derivation of some necessary and sufficient conditions for the existence of positive solutions to a singular second-order system of dynamic equations with Dirichlet boundary conditions. The results are obtained by employing fixed-point theorems and the method of lower and upper solutions.
\end{abstract}

MSC: 34B16; 34K10; 39A 13

Keywords: time scales; boundary value problem in dynamic equations; upper and lower solutions

\section{Introduction}

The main purpose of this paper is to establish existence results for the second-order Dirichlet system

$(P) \quad\left\{\begin{array}{l}x_{i}^{\Delta \Delta}=f_{i}\left(t, x^{\sigma}(t)\right), \quad t \in\left(J^{\kappa}\right)^{o}, \\ x_{i}(a)=A_{i}, \quad x_{i}\left(\sigma^{2}(b)\right)=B_{i}, \quad i=1,2, \ldots, n\end{array}\right.$

with $f=\left(f_{1}, \ldots, f_{n}\right), i=1,2, \ldots, n$, where $f_{i}:\left(J^{\kappa}\right)^{o} \times \mathcal{A} \rightarrow \mathbb{R}, \mathcal{A} \subset \mathbb{R}^{n}$ and $J$ is a time scale interval. The nonlinearity $f_{i}(t, x)$ may be singular at $x_{i}, i=1, \ldots, n$ and/or $t$.

Stefan Hilger [1] introduced the notion of time scale in 1988 in order to unify the theory of continuous and discrete calculus. The time scales approach not only unifies differential and difference equations, but also solves some other problems such as a mix of stopstart and continuous behaviors [2,3] powerfully. Nowadays the theory on time scales has been widely applied to several scientific fields such as biology, heat transfer, stock market, wound healing and epidemic models.

Under the general form of problem $(P)$, it included the Emden-Fowler equation which arises, for example, in astrophysics in relation to the stellar structure (gaseous dynamics). In this case, the fundamental problem is to investigate the equilibrium configuration of the mass of spherical clouds of gas. It also arises in gas dynamics and fluid mechanics. The solutions of physical interest in this context are bounded non-oscillatory and possess a positive zero. It is also encountered in the relativistic mechanics and nuclear physics; and in chemically reacting systems: in the theory of diffusion and reaction this equation appears as governing the concentration $u$ of a substance which disappears by an isothermal reaction at each point of a slab of catalyst. We refer to Wong [4] for a general historical overview of this equation.

o 2012 Lago et al.; licensee Springer. This is an Open Access article distributed under the terms of the Creative Commons Attribution License (http://creativecommons.org/licenses/by/2.0), which permits unrestricted use, distribution, and reproduction in any medium, provided the original work is properly cited. 
Many works on this system have been written in the continuous case. We can cite, among others, [5, 6] or [7] for $n=1$ or [8] for $n=2$. On the discrete case, we find the book [9] which studies the oscillation properties of the solutions of different difference equations. For the specific problem $u^{\Delta \Delta}(t)+p(t) u^{\gamma}(\sigma(t))=0$, where $p \geq 0$ and $\gamma$ is the quotient of odd positive numbers, oscillation properties were also studied in [10].

Regarding time scales, some results on the existence and uniqueness of classical solutions or solutions in the sense of distribution for $n=1$ can be found in the articles [11-13] and [14]. Considering classical solutions, oscillation properties have also been studied in works such as [15] (with delay) or [16].

In the present paper, we present some results on time scales considering classical solutions which generalize the ones from the continuous case. The remainder of the paper is organized as follows. In Section 2, we state some existence results supposing the existence of a pair of lower and upper solutions and employing the Schauder fixed-point theorem. In Section 3, we shall give a necessary and sufficient condition for the existence of positive solutions of singular boundary value problem $(P)$ by constructing a lower solution.

\section{Lower and upper solutions method}

Let $\mathbb{T}$ be an arbitrary time scale. We assume that $\mathbb{T}$ has the topology that it inherits from the standard topology on $\mathbb{R}$. See [17] for general theory on time scales.

Let $a, b \in \mathbb{T}$ be such that $a<\rho(b)$. If a is a right-dense point, we consider $J=\left(a, \sigma^{2}(b)\right]_{\mathbb{T}}$, $J^{\kappa}=(a, \sigma(b)]_{\mathbb{T}}$ and $\left(J^{\kappa}\right)^{o}=(a, \sigma(b))_{\mathbb{T}}$. In the other case, $J=\left[a, \sigma^{2}(b)\right]_{\mathbb{T}}, J^{\kappa}=[a, \sigma(b)]_{\mathbb{T}}$ and $\left(J^{\kappa}\right)^{o}=[a, \sigma(b))_{\mathbb{T}}$.

The problem we will consider in this section is

$(P) \quad\left\{\begin{array}{l}x_{i}^{\Delta \Delta}=f_{i}\left(t, x^{\sigma}(t)\right), \quad t \in\left(J^{\kappa}\right)^{o}, \\ x_{i}(a)=A_{i}, \quad x_{i}\left(\sigma^{2}(b)\right)=B_{i}, \quad i=1,2, \ldots, n .\end{array}\right.$

with $f=\left(f_{1}, \ldots, f_{n}\right), i=1,2, \ldots, n$, where $f_{i}:\left(J^{\kappa}\right)^{o} \times \mathcal{A} \rightarrow \mathbb{R}, \mathcal{A} \subset \mathbb{R}^{n}$.

We say that $f$ verifies the hypothesis $\left(\mathrm{H}_{1}\right)$ if for every $i=1,2, \ldots, n$, the following conditions are satisfied:

(i) For every $x \in \mathcal{A}, f_{i}(\cdot, x) \in C_{r d}\left(\left(J^{\kappa}\right)^{o}\right)$,

(ii) $f_{i}(t, \cdot)$ is continuous on $\mathcal{A}$ uniformly in $t \in\left(J^{\kappa}\right)^{o}$.

For convenience, we denote

$$
E=\left\{g \in C_{r d}\left(\left(J^{\kappa}\right)^{o}, \mathbb{R}^{+}\right): \int_{a}^{\sigma(b)}(\sigma(s)-a)\left(\sigma^{2}(b)-s\right) g(s) \Delta s<+\infty\right\} .
$$

We say that $f$ satisfies the condition $\left(\mathrm{H}_{2}\right)$ on $\mathcal{B} \subset\left(J^{\kappa}\right)^{o} \times \mathcal{A}$ if for $i=1,2, \ldots, n$ there exists a function $h_{i} \in E$ such that

$\left(\mathrm{H}_{2}\right) \quad\left|f_{i}(t, x)\right| \leq h_{i}(t), \forall(t, x) \in \mathcal{B}$.

Definition 2.1 A solution of $(P)$ is a function $x=\left(x_{1}, \ldots, x_{n}\right)$, with $x_{i} \in C_{r d}^{2}\left((a, b)_{\mathbb{T}}\right)$ for all $i=1, \ldots, n$ such that $x(t) \in \mathcal{A}$ for all $t \in\left[a, \sigma^{2}(b)\right]_{\mathbb{T}}$, which satisfies $(P)$ for each $t \in\left(J^{\kappa}\right)^{o}$ and $i=1, \ldots, n$, where

$$
C_{r d}^{2}\left((a, b)_{\mathbb{T}}\right)=\left\{y \in C\left(\left[a, \sigma^{2}(b)\right]_{\mathbb{T}}\right), y^{\Delta \Delta}:\left(J^{\kappa}\right)^{o} \rightarrow \mathbb{R} \text {, and } y^{\Delta \Delta} \in C_{r d}\left((a, b)_{\mathbb{T}}\right)\right\} .
$$


Definition 2.2 We say that $\alpha=\left(\alpha_{1}, \ldots, \alpha_{n}\right)$, with $\alpha_{i} \in C_{r d}^{2}\left((a, b)_{\mathbb{T}}\right)$, is a lower solution of $(P)$ if $\alpha(t) \in \mathcal{A}$ for all $t \in\left[a, \sigma^{2}(b)\right]_{\mathbb{T}}$ and

$$
\begin{aligned}
& -\alpha^{\Delta \Delta}(t) \leq f\left(t, \alpha^{\sigma}(t)\right), \quad t \in\left(J^{\kappa}\right)^{o}, \\
& \alpha(a) \leq A, \quad \alpha\left(\sigma^{2}(b)\right) \leq B .
\end{aligned}
$$

An upper solution $\beta=\left(\beta_{1}, \ldots, \beta_{n}\right)$ of $(P)$ is defined similarly by reversing the previous inequalities.

We have the following result.

Theorem 2.1 Let $\alpha$ and $\beta$ be, respectively, a lower and upper solution for problem $(P)$ such that $\alpha \leq \beta$ on $\left[a, \sigma^{2}(b)\right]_{\mathbb{T}}$. Iff satisfies $\left(\mathrm{H}_{1}\right)$ and the condition $\left(\mathrm{H}_{2}\right)$ on

$$
\mathcal{D}_{\alpha}^{\beta}=\left\{(t, x) \in\left(J^{\kappa}\right)^{o} \times \mathbb{R}: \alpha^{\sigma}(t) \leq x \leq \beta^{\sigma}(t)\right\}
$$

and

$\left(\mathrm{H}_{3}\right)$ For $t \in[a, b]_{T}$ and $x \in \mathcal{A}: \alpha(t) \leq x \leq \beta(t) \Rightarrow$

$$
f_{i}(t, \alpha(t)) \leq f_{i}(t, x) \leq f_{i}(t, \beta(t))
$$

for all $i=1, \ldots, n$.

Then problem $(P)$ has at least one solution $x$ such that $\alpha \leq x \leq \beta$ on $\left[a, \sigma^{2}(b)\right]_{\mathbb{T}}$.

Proof We consider the following modified problem:

$$
\left(P_{m}\right) \quad\left\{\begin{array}{l}
x_{i}^{\Delta \Delta}(t)=-f_{i}^{*}\left(t, x^{\sigma}(t)\right), \quad t \in\left(J^{\kappa}\right)^{o}, \\
x_{i}(a)=A_{i}, \quad x_{i}\left(\sigma^{2}(b)\right)=B_{i}, \quad i=1,2, \ldots, n
\end{array}\right.
$$

with

$$
f_{i}^{*}(t, x)=f_{i}\left(t, d_{i}(t, x)\right)+\frac{d_{i}(t, x)-x_{i}}{1+\left|d_{i}(t, x)-x_{i}\right|}
$$

where $d=\left(d_{1}, \ldots, d_{n}\right)$ and $d_{i}:\left[a, \sigma^{2}(b)\right]_{\mathbb{T}} \times \mathcal{A} \rightarrow \mathbb{R}$ is defined

$$
d_{i}(t, x)= \begin{cases}\alpha_{i}^{\sigma}(t), & \text { if } x_{i}<\alpha_{i}^{\sigma}(t) \\ x_{i}, & \text { if } \alpha_{i}^{\sigma}(t) \leq x_{i} \leq \beta_{i}^{\sigma}(t) \\ \beta_{i}^{\sigma}(t), & \text { if } \beta_{i}^{\sigma}(t) \leq x_{i} .\end{cases}
$$

We can prove that $d_{i}(t, \cdot)$ is continuous on $\mathcal{A}$ uniformly in $t$ and $d_{i}(\cdot, x) \in C_{r d}\left(\left[a, \sigma^{2}(b)\right]_{\mathbb{T}}\right)$ for every $x \in \mathcal{A}$. Hence, the function $p: \mathcal{D}_{\alpha}^{\beta} \rightarrow \mathcal{D}_{\alpha}^{\beta}, p(t, x)=(t, d(t, x))$ verified for each $\left.x \in \mathcal{A}, p(\cdot, x) \in C_{r d}\left(J^{\kappa}\right)^{o}\right)$.

Due to the hypothesis, it is easy to see that $\left(\mathrm{H}_{1}\right)$ is satisfied and that there exist $h_{i}^{*} \in E$ such that $\left(\mathrm{H}_{2}\right)$ holds for the function $f^{*}$. 
Note that if $u$ is a solution of $\left(P_{m}\right)$ such that $\alpha \leq u \leq \beta$ on $\left[a, \sigma^{2}(b)\right]_{\mathbb{T}}$, then $u$ is a solution of $(P)$.

To show that any solution $u$ of $\left(P_{m}\right)$ is between $\alpha$ and $\beta$, suppose that there exists $i=$ $1, \ldots, n$ and $t^{*} \in\left[a, \sigma^{2}(b)\right]_{\mathbb{T}}$ such that $v_{i}\left(t^{*}\right)=\alpha_{i}(t)-u_{i}(t)>0$. As $v_{i}(a) \leq 0$ and $v_{i}\left(\sigma^{2}(b)\right) \leq 0$, then there exists $t_{0} \in\left(a, \sigma^{2}(b)\right)_{\mathbb{T}}$ with

$$
v_{i}\left(t_{0}\right)=\max \left\{v_{i}(t), t \in\left[a, \sigma^{2}(b)\right]_{\mathbb{T}}\right\}>0,
$$

and $v_{i}(t)<v_{i}\left(t_{0}\right)$ for $t \in\left(t_{0}, \sigma^{2}(b)\right]_{\mathbb{T}}$. The point $t_{0}$ is not simultaneously left-dense and right-scattered (see Theorem 2.1 in [12]). This implies that $(\sigma \circ \rho)\left(t_{0}\right)=t_{0}$, and we have that $v_{i}^{\Delta \Delta}\left(\rho\left(t_{0}\right)\right) \leq 0$ (see [12]), so given that $\alpha^{\sigma}(t) \leq d\left(t, u^{\sigma}(t)\right)$,

$$
\begin{aligned}
-v_{i}^{\Delta \Delta}\left(\rho\left(t_{0}\right)\right)= & u_{i}^{\Delta \Delta}\left(\rho\left(t_{0}\right)\right)-\alpha_{i}^{\Delta \Delta}\left(\rho\left(t_{0}\right)\right) \\
\leq & -f_{i}\left(\rho\left(t_{0}\right), d\left(\rho\left(t_{0}\right), u^{\sigma}\left(\rho\left(t_{0}\right)\right)\right)\right) \\
& -\frac{d_{i}\left(\rho\left(t_{0}\right), u^{\sigma}\left(\rho\left(t_{0}\right)\right)\right)-x_{i}^{\sigma}\left(\rho\left(t_{0}\right)\right)}{1+\left|d_{i}\left(\rho\left(t_{0}\right), u^{\sigma}\left(\rho\left(t_{0}\right)\right)\right)-x_{i}^{\sigma}\left(\rho\left(t_{0}\right)\right)\right|}+f\left(\rho\left(t_{0}\right), \alpha^{\sigma}\left(\rho\left(t_{0}\right)\right)\right) \\
= & -\frac{\alpha\left(t_{0}\right)-u\left(t_{0}\right)}{1+\left(\alpha\left(t_{0}\right)-u\left(t_{0}\right)\right)}<0 .
\end{aligned}
$$

So, $v^{\Delta \Delta}\left(\rho\left(t_{0}\right)\right)>0$, that is a contradiction. And so we have proved that $v(t) \leq 0$ for each $t \in\left[a, \sigma^{2}(b)\right]_{\mathbb{T}}$.

Analogously, it can be proved that $u(t) \leq \beta(t)$ for all $t \in\left[a, \sigma^{2}(b)\right]_{\mathbb{T}}$.

We only need to prove that problem $\left(P_{m}\right)$ has at least one solution.

Consider now the operator $N: C\left(\left[a, \sigma^{2}(b)\right]_{\mathbb{T}}\right) \rightarrow C\left(\left[a, \sigma^{2}(b)\right]_{\mathbb{T}}\right)$ defined by

$$
N u(t)=\phi(t)+\int_{a}^{\sigma(b)} G(t, s) f^{*}\left(s, u^{\sigma}(s)\right) \Delta s
$$

for each $t \in\left[a, \sigma^{2}(b)\right]_{\mathbb{T}}$, where (see [17])

$$
G(t, s)=\frac{1}{\sigma^{2}(b)-a} \begin{cases}(t-a)\left(\sigma^{2}(b)-\sigma(s)\right), & t \leq s, \\ (\sigma(s)-a)\left(\sigma^{2}(b)-t\right), & \sigma(s) \leq t,\end{cases}
$$

is Green's function of the problem

$$
\left\{\begin{array}{l}
-x^{\Delta \Delta}=0 \\
x(a)=x\left(\sigma^{2}(b)\right)=0
\end{array}\right.
$$

and for $t \in\left[a, \sigma^{2}(b)\right]_{\mathbb{T}}$,

$$
\phi(t)=A+\frac{B-A}{\sigma^{2}(b)-a}(t-a)
$$

is the solution of $-x^{\Delta \Delta}=0$ such that $\phi(a)=A$ and $\phi\left(\sigma^{2}(b)\right)=B$.

Clearly, $G(t, s)>0$ on $\left(a, \sigma^{2}(b)\right)_{\mathbb{T}} \times\left(a, \sigma^{2}(b)\right)_{\mathbb{T}}, G(t, \cdot)$ is rd-continuous on $[a, \sigma(b)]_{\mathbb{T}}$ and $G(\cdot, s)$ is continuous on $\left[a, \sigma^{2}(b)\right]_{\mathbb{T}}$. 
The function $N u$ defined by (2.1) belongs to $C\left(\left[a, \sigma^{2}(b)\right]_{\mathbb{T}}\right)$ because $f^{\prime \prime}$ satisfies the conditions $\left(\mathrm{H}_{1}\right)$ and $\left(\mathrm{H}_{2}\right)$ on $\left(J^{\kappa}\right)^{o} \times \mathbb{R}$ and $G(t, s) \leq s(1-s)$ for each $t, s \in\left[a, \sigma^{2}(b)\right]_{\mathbb{T}}$.

It is obvious that $u \in C\left(\left[a, \sigma^{2}(b)\right]_{\mathbb{T}}\right)$ is a solution of $\left(P_{m}\right)$ if and only if $u=N u$. So, the problem now is to ensure the existence of fixed points of $N$.

First of all, $N$ is well defined, continuous and $N\left(C\left(\left[a, \sigma^{2}(b)\right]_{\mathbb{T}}\right)\right)$ is a bounded set. The existence of a fixed point of $N$ follows from the Schauder fixed-point theorem once we have checked that $N\left(C\left(\left[a, \sigma^{2}(b)\right]_{\mathbb{T}}\right)\right)$ is relatively compact, that using the Ascoli-Arzela theorem is equivalent to proving that $N\left(C\left(\left[a, \sigma^{2}(b)\right]_{\mathbb{T}}\right)\right)$ is an equicontinuous family.

Let $h^{*} \in E$ be the function related to $f^{*}$ by the condition $\left(\mathrm{H}_{2}\right)$. We compute the first derivative of $\mathrm{Nu}$ using Theorem 1.117 of [17]:

$$
\begin{aligned}
\left|(N u)^{\Delta}(t)\right|= & \frac{1}{\sigma^{2}(b)-a} \mid(B-A)-\int_{a}^{t}(\sigma(s)-a) f^{*}\left(s, u^{\sigma}(s)\right) \Delta s \\
& +\int_{t}^{\sigma(b)}\left(\sigma^{2}(b)-\sigma(s)\right) f^{*}\left(s, u^{\sigma}(s)\right) \Delta s \mid \\
\leq & \frac{1}{\sigma^{2}(b)-a}\left(|B-A|+\int_{a}^{t}(\sigma(s)-a) h^{\prime \prime}(s) \Delta s\right. \\
& \left.+\int_{t}^{\sigma(b)}\left(\sigma^{2}(b)-\sigma(s)\right) h^{*}(s) \Delta s\right) \\
:= & \frac{1}{\sigma^{2}(b)-a}(|B-A|+\lambda(t)) .
\end{aligned}
$$

Finally, it is enough to check that $\lambda \in L^{1}\left(\left(J^{\kappa}\right)^{o}\right)$. Using integration by parts, we obtain

$$
\begin{aligned}
\int_{a}^{\sigma(b)}|\lambda(s)| \Delta s= & \int_{a}^{\sigma(b)} \lambda(s) \Delta s \\
= & \lim _{r \rightarrow \sigma(b)^{-}} \int_{a}^{r}\left(\int_{a}^{t}(\sigma(s)-a) h^{*}(s) \Delta s\right) \Delta t \\
& +\lim _{r \rightarrow a^{+}} \int_{r}^{\sigma(b)}\left(\int_{t}^{\sigma(b)}\left(\sigma^{2}(b)-\sigma(s)\right) h^{*}(s) \Delta s\right) \Delta t \\
= & 2 \int_{a}^{\sigma(b)}(\sigma(s)-a)\left(\sigma^{2}(b)-\sigma(s)\right) h^{*}(s) \Delta s \\
& -\lim _{r \rightarrow \sigma(b)^{-}}\left(\sigma^{2}(b)-r\right) \int_{a}^{r}(\sigma(s)-a) h^{*}(s) \Delta s \\
& -\lim _{r \rightarrow a^{+}}(r-a) \int_{r}^{\sigma(b)}\left(\sigma^{2}(b)-\sigma(s)\right) h^{*}(s) \Delta s<+\infty
\end{aligned}
$$

due to $h^{*} \in E$ and the fact

$$
\begin{aligned}
& \left(\sigma^{2}(b)-r\right) \int_{a}^{r}(\sigma(s)-a) h^{*}(s) \Delta s \leq \int_{a}^{\sigma(b)}(\sigma(s)-a)\left(\sigma^{2}(b)-\sigma(s)\right) h^{*}(s) \Delta s, \\
& (r-a) \int_{r}^{\sigma(b)}\left(\sigma^{2}(b)-\sigma(s)\right) h^{*}(s) \Delta s \leq \int_{a}^{\sigma(b)}(\sigma(s)-a)\left(\sigma^{2}(b)-\sigma(s)\right) h^{*}(s) \Delta s .
\end{aligned}
$$

And so the result is proved. 
Remark 2.1 The above theorem is also true if we change $\left(\mathrm{H}_{3}\right)$ by

$\left(\overline{\mathrm{H}}_{3}\right)$ For $t \in[a, b]_{T}$ and $x, y \in \mathcal{A}$, there exists $M>0$ such that

$$
f_{i}(t, x)-f_{i}(t, y) \leq M(x-y)
$$

Remark 2.2 The existence of a lower solution and an upper solution with $0<\alpha \leq \beta$ can be obtained through conditions of $f_{i}$. For instance, if $f_{i} \in C_{r d}(J)$ and $f_{i}$ is bounded, the existence holds.

\section{Existence of a positive solution}

Consider the problem

$$
\left(P_{0}\right) \quad \begin{cases}-x_{i}^{\Delta \Delta}(t)=f_{i}\left(t, x^{\sigma}(t)\right), & t \in\left(J^{\kappa}\right)^{o}, \\ x_{i}(a)=x_{i}\left(\sigma^{2}(b)\right)=0, & i=0, \ldots, n .\end{cases}
$$

We will deduce the existence of a solution to $\left(P_{0}\right)$ by supposing that the following hypotheses hold:

$\left(\widetilde{\mathrm{H}}_{1}\right)$ For every $i=1, \ldots, n, f_{i}:\left(J^{\kappa}\right)^{o} \times \mathcal{A} \rightarrow[0,+\infty)$, where $\mathcal{A} \subset(0,+\infty) \times \cdots^{n} \times(0,+\infty)$, verifies

(i) For every $x \in \mathcal{A}, f_{i}(\cdot, x) \in C_{r d}\left(\left(J^{\kappa}\right)^{o}\right)$,

(ii) $f_{i}(t, \cdot)$ is continuous on $\mathcal{A}$ uniformly in $t \in\left(J^{\kappa}\right)^{o}$.

$\left(\widetilde{\mathrm{H}}_{2}\right)$ For every $i=1, \ldots, n$, and $j=1, \ldots, n$, there exist constants $\lambda_{i j}, \mu_{i j}$, with $-\infty<\lambda_{i j}<\mu_{i j}<$ $1, \lambda_{i i}<0<\mu_{i i}<1, \mu_{i j}<0$ if $i \neq j$, such that if $0<c \leq 1$, then

$$
c^{\mu_{i j}} f_{i}\left(t, x_{1}, \ldots, x_{n}\right) \leq f_{i}\left(t, x_{1}, \ldots, c x_{j}, \ldots, x_{n}\right) \leq c^{\lambda_{i j}} f_{i}\left(t, x_{1}, \ldots, x_{n}\right)
$$

for each $t \in\left(J^{\kappa}\right)^{o}$ and $x \in \mathcal{A}$.

Remark 3.1 If $c \geq 1$ for every $i=1, \ldots, n$,

$$
c^{\lambda_{i j}} f_{i}\left(t, x_{1}, \ldots, x_{n}\right) \leq f_{i}\left(t, x_{1}, \ldots, c x_{j}, \ldots, x_{n}\right) \leq c^{\mu_{i j}} f_{i}\left(t, x_{1}, \ldots, x_{n}\right)
$$

for each $t \in\left(J^{\kappa}\right)^{o}$ and $x \in \mathcal{A}$.

We consider solutions to the problem.

Definition 3.1 A positive solution of type 1 of $\left(P_{0}\right)$ is a function $x=\left(x_{1}, \ldots, x_{n}\right)$, with $x_{i} \in$ $C_{r d}^{2}\left((a, b)_{\mathbb{T}}\right)$ for all $i=1, \ldots, n$ such that $x(t) \in \mathcal{A}$ and $x_{i}(t)>0$ for all $t \in\left[a, \sigma^{2}(b)\right]_{\mathbb{T}}$, which satisfies the equalities on $\left(P_{0}\right)$ for each $t \in\left(J^{\kappa}\right)^{o}$ and $i=1, \ldots, n$, and the following limits exist and are finite:

$$
\lim _{t \rightarrow a^{+}} x_{i}^{\Delta}(t) \text { and } \lim _{t \rightarrow \sigma(b)^{-}} x_{i}^{\Delta}(t) .
$$


Definition 3.2 We say that $\alpha \in C_{r d}^{2}$ is a lower solution of $\left(P_{0}\right)$ if for each $i=1,2, \ldots, n$ we have

$$
\left\{\begin{array}{l}
-\alpha_{i}^{\Delta \Delta}(t) \leq f_{i}\left(t, x^{\sigma}(t)\right), \quad t \in\left(J^{\kappa}\right)^{o} \\
\alpha_{i}(a)=\alpha_{i}\left(\sigma^{2}(b)\right)=0
\end{array}\right.
$$

Similarly, $\beta \in C_{r d}^{2}$ is called an upper solution of $\left(P_{0}\right)$ if for each $i=1,2, \ldots, n$,

$$
\left\{\begin{array}{l}
-\beta_{i}^{\Delta \Delta}(t) \geq f_{i}\left(t, x^{\sigma}(t)\right), \quad t \in\left(J^{\kappa}\right)^{o} \\
\beta_{i}(a)=\beta_{i}\left(\sigma^{2}(b)\right)=0
\end{array}\right.
$$

Lemma 3.1 Suppose that $\left(\widetilde{\mathrm{H}}_{1}\right)$ and $\left(\tilde{\mathrm{H}}_{2}\right)$ hold. If $x$ is a positive solution of type 1 of $\left(P_{0}\right)$, then for each $i=1, \ldots, n$, there are constants $I_{i 1}, I_{i 2}, 0<I_{i 1}<I_{i 2}$ such that

$$
I_{i 1} e(t) \leq x_{i}(t) \leq e(t) I_{i 2}
$$

where $e(t)=\frac{(t-a)\left(\sigma^{2}(b)-t\right)}{\sigma^{2}(b)-a}$.

Proof Integrate the equations of $\left(P_{0}\right)$ in $\left(J^{\kappa}\right)^{o}$ for $i=1, \ldots, n$

$$
\begin{aligned}
-\int_{a}^{\sigma(b)} f_{i}\left(s, x^{\sigma}(s)\right) \Delta s & =\int_{a}^{\sigma(b)} x_{i}^{\Delta \Delta}(s) \Delta s \\
& =\lim _{t \rightarrow \sigma(b)^{-}} x_{i}^{\Delta}(t)-\lim _{t \rightarrow a^{+}} x_{i}^{\Delta}(t)<+\infty .
\end{aligned}
$$

From (2.2), we have

$$
x_{i}(t)=\int_{a}^{\sigma(b)} G(t, s) f_{i}\left(s, x^{\sigma}(s)\right) \Delta s \leq \frac{(t-a)\left(\sigma^{2}(b)-t\right)}{\sigma^{2}(b)-a} \int_{a}^{\sigma(b)} f_{i}\left(s, x^{\sigma}(s)\right) \Delta s .
$$

Since

$$
\begin{aligned}
& \int_{a}^{t} \frac{(s-a)\left(\sigma^{2}(b)-\sigma(s)\right)(t-a)\left(\sigma^{2}(b)-t\right)}{\left(\sigma^{2}(b)-a\right)^{2}} f_{i}\left(s, x^{\sigma}(s)\right) \Delta s \\
& \quad \leq \int_{a}^{t}(\sigma(s)-a)\left(\sigma^{2}(b)-t\right) f_{i}\left(s, x^{\sigma}(s)\right) \Delta s,
\end{aligned}
$$

and

$$
\begin{gathered}
\int_{t}^{\sigma(b)} \frac{(s-a)\left(\sigma^{2}(b)-\sigma(s)\right)(t-a)\left(\sigma^{2}(b)-t\right)}{\left(\sigma^{2}(b)-a\right)^{2}} f_{i}\left(s, x^{\sigma}(s)\right) \Delta s \\
\quad \leq \int_{t}^{\sigma(b)}(t-a)\left(\sigma^{2}(b)-\sigma(s)\right) f_{i}\left(s, x^{\sigma}(s)\right) \Delta s,
\end{gathered}
$$

we have

$$
\frac{(t-a)\left(\sigma^{2}(b)-t\right)}{\left(\sigma^{2}(b)-a\right)^{2}} \int_{a}^{\sigma(b)} \frac{(s-a)\left(\sigma^{2}(b)-\sigma(s)\right)}{\left(\sigma^{2}(b)-a\right)} f_{i}\left(s, x^{\sigma}(s)\right) \Delta s \leq x_{i}(t) .
$$


Thus, if we consider

$$
\begin{aligned}
I_{i 1} & =\frac{1}{\left(\sigma^{2}(b)-a\right)} \int_{a}^{\sigma(b)} \frac{(s-a)\left(\sigma^{2}(b)-\sigma(s)\right)}{\left(\sigma^{2}(b)-a\right)} f_{i}\left(s, x^{\sigma}(s)\right) \Delta s, \\
I_{i 2} & =\int_{a}^{\sigma(b)} f_{i}\left(s, x^{\sigma}(s)\right) \Delta s,
\end{aligned}
$$

it verifies

$$
I_{i 1} e(t) \leq x_{i}(t) \leq e(t) I_{i 2}
$$

Lemma 3.2 If $\alpha$ and $\beta$ are lower and upper solutions of problem $\left(P_{0}\right)$ such that $0<\alpha(t) \leq$ $\beta(t)$ for $t \in\left(J^{\kappa}\right)^{o}$, and $\left(\widetilde{\mathrm{H}}_{1}\right)$ and $\left(\mathrm{H}_{3}\right)$ or $\left(\overline{\mathrm{H}}_{3}\right)$ hold, then problem $\left(P_{0}\right)$ has a solution $x$ such that

$$
\alpha \leq x \leq \beta
$$

If in addition there exists a function $h(t)=\left(h_{1}(t), \ldots, h_{n}(t)\right)$ with $h_{i} \in L^{1}\left(\left(J^{\kappa}\right)^{o}\right)$ such that

$$
\left|f_{i}(t, x)\right| \leq h_{i}(t), \quad \forall t \in\left(J^{\kappa}\right)^{o} \quad \text { and } \quad \alpha_{i}(t) \leq x_{i} \leq \beta_{i}(t), \quad i=1, \ldots, n,
$$

then the solution $x$ is a positive solution of type 1.

Proof Let us consider $\left\{a_{k}\right\}_{k \geq 1},\left\{b_{k}\right\}_{k \geq 1} \subset\left(J^{\kappa}\right)^{o}$ to be two sequences such that $\left\{a_{k}\right\}_{k \geq 1} \subset$ $(a,(a+\sigma(b)) / 2)_{\mathbb{T}}$ is strictly decreasing to $a$ if $a=\sigma(a)$, and $a_{k}=a$ for all $k \geq 1$ if $a<$ $\sigma(a)$, and $\left\{b_{k}\right\}_{k \geq 1} \subset((a+\sigma(b)) / 2, \sigma(b))_{\mathbb{T}}$ is strictly increasing to $\sigma(b)$ if $\rho(\sigma(b))=\sigma(b)$, $b_{k}=\rho(\sigma(b))$ for all $k \geq 1$ if $\rho(\sigma(b))<\sigma(b)$.

We denote $D_{k}:=\left[a_{k}, b_{k}\right]_{\mathbb{T}} \subset\left(J^{\kappa}\right)^{o}, k \geq 1$, and let $\left\{r_{i 1}^{k}\right\},\left\{r_{i 2}^{k}\right\}$ be sequences so that

$$
\begin{aligned}
& \alpha_{i}\left(a_{k}\right) \leq r_{i 1}^{k} \leq \beta_{i}\left(a_{k}\right), \\
& \alpha_{i}\left(b_{k}\right) \leq r_{i 2}^{k} \leq \beta_{i}\left(k_{k}\right) .
\end{aligned}
$$

For each $x \in \mathcal{A}$, define

$$
f_{k i}^{*}(\cdot, x): D_{k} \rightarrow[0, \infty)
$$

for all $k \in \mathbb{N}, k \geq 1$ and $i=1, \ldots, n$, as

$$
f_{k i}^{*}(t, x)=f_{i}(t, d(t, x))+\frac{d_{i}(t, x)-x_{i}}{1+\left|d_{i}(t, x)-x_{i}\right|} .
$$

Consider the problems

$\left(P_{k}\right) \quad\left\{\begin{array}{l}x_{i}^{\Delta \Delta}(t)=-f_{k i}^{*}\left(t, x^{\sigma}(t)\right), \quad t \in D_{k}, \\ x_{i}\left(a_{k}\right)=r_{i 1}^{k}, \quad x_{i}\left(\sigma^{2}\left(b_{k}\right)\right)=r_{i 2}^{k}, \quad i=1, \ldots, n .\end{array}\right.$ 
Due to the hypothesis $f_{i}, i=1, \ldots, n$, by Theorem 2.1, we can ensure that there exists a solution $\left(x_{k 1}, \ldots, x_{k n}\right)$ with $x_{k i} \in C_{r d}\left[a_{k}, \sigma^{2}\left(b_{k}\right)\right]$ such that

$$
\alpha_{i}(t) \leq x_{k i}(t) \leq \beta_{i}(t)
$$

with $t \in\left[a_{k}, b_{k}\right]$. Since $\left[a_{1}, b_{1}\right] \subset\left[a_{k}, b_{k}\right]$ for $k \in \mathbb{N}$, there exists $t_{k} \in\left[a_{1}, b_{1}\right]$ such that

$$
\left|x_{k i}^{\Delta}\left(t_{k}\right)\right| \leq \frac{\left|x_{k i}\left(b_{1}\right)-x_{k i}\left(a_{1}\right)\right|}{\left|b_{1}-a_{1}\right|} \leq \frac{2\left(\beta_{i}\left(b_{1}\right)-\beta_{i}\left(a_{1}\right)\right)}{\left|b_{1}-a_{1}\right|} .
$$

Thus, we can find a sequence $\left\{t_{k}\right\}$ which converges to $t_{0} \in\left[a_{k}, b_{k}\right]$ for $k \in \mathbb{N}$, satisfying

$$
x_{k i}\left(t_{k}\right) \rightarrow x_{0 i} \in\left[\alpha_{i}\left(t_{0}\right), \beta_{i}\left(t_{0}\right)\right],
$$

and

$$
x_{k i}^{\Delta}\left(t_{k}\right) \rightarrow x_{0 i}^{\Delta}
$$

for $i=1, \ldots, n$ when $k \rightarrow \infty$.

We note that $x_{k i}$ is the solution of

$$
y_{i}^{\Delta \Delta}(t)=-f_{i}\left(t, y^{\sigma}(t)\right)
$$

with $y_{i}\left(t_{k}\right)=x_{k i}\left(t_{k}\right)$ and $y_{i}^{\Delta}\left(t_{k}\right)=x_{k i}^{\Delta}\left(t_{k}\right)$.

Hence, due to an adaptation of Theorem 3.2 in [18] and by existence theorems, we can find a solution of the problem

$$
x_{i}^{\Delta \Delta}(t)=-f_{i}\left(t, x^{\sigma}(t)\right), \quad x_{i}\left(t_{0}\right)=x_{0 i}, \quad x_{i}^{\Delta}\left(t_{0}\right)=x_{0 i}^{\Delta}, \quad i=1, \ldots, n .
$$

This solution is defined in a maximal interval $W$, and we can find at least one sequence $\left\{x_{k}(t)\right\}$ that converges uniformly to $x(t)$ in the compact subintervals of $W$.

On the other hand, $\bigcup_{k=1}^{\infty}\left[a_{k}, b_{k}\right]=\left(J^{\kappa}\right)^{o}$ and $\alpha_{i}(t) \leq x_{k i}(t) \leq \beta_{i}(t)$ for $t \in\left[a_{k}, b_{k}\right]$, then $x$ is defined in $\left(J^{\kappa}\right)^{o}$ and $\alpha(t) \leq x(t) \leq \beta(t)$ for all $t \in\left(J^{\kappa}\right)^{o}$. From the conditions on $\alpha$ and $\beta$ on the boundary, it follows that

$$
x(a)=x\left(\sigma^{2}(b)\right)=0,
$$

so that $x$ is a solution of problem $\left(P_{0}\right)$.

Suppose there exists a function $h(t)=\left(h_{1}(t), \ldots, h_{n}(t)\right)$ with $\left.h_{i} \in L^{1}\left(J^{\kappa}\right)^{o}\right)$ such that

$$
\left|f_{i}(t, x)\right| \leq h_{i}(t), \quad \forall t \in\left(J^{\kappa}\right)^{o} \quad \text { and } \quad \alpha_{i}(t) \leq x_{i} \leq \beta_{i}(t), \quad i=1, \ldots, n,
$$

then we can assume that $\left|x_{i}^{\Delta \Delta}(t)\right| \leq h_{i}(t), i=1, \ldots, n$, which implies that $x_{i}^{\Delta \Delta}$ is absolutely integrable on $[a, \sigma(b)]_{\mathbb{T}}$ and $x_{i}^{\Delta} \in C[a, \sigma(b)], i=1, \ldots, n$, so $x$ is a positive solution of type 1. 
Theorem 3.1 Suppose that $\left(\widetilde{\mathrm{H}}_{1}\right),\left(\widetilde{\mathrm{H}}_{2}\right)$ and $\left(\mathrm{H}_{3}\right)$ or $\left(\overline{\mathrm{H}_{3}}\right)$ hold. There exists a positive solution of type 1 if and only if the following conditions hold:

$$
0<\int_{a}^{\sigma(b)} f_{i}\left(s, E^{\sigma}(s)\right) \Delta s<\infty,
$$

for all $i=1, \ldots, n$, where $E(t)=(e(t), \ldots, e(t))$.

Proof Necessity. Suppose that there exists $x=\left(x_{1}, \ldots, x_{n}\right)$ positive solutions of type 1 of $\left(P_{0}\right)$. By Lemma 3.1, there are constants $I_{i 1}, I_{i 2}, 0<I_{i 1}<I_{i 2}$ for each $i=1, \ldots, n$ such that

$$
I_{i 1} e(t) \leq x_{i}(t) \leq e(t) I_{i 2}
$$

Let $K>0$ such that $K I_{i 2} \leq 1, \frac{1}{K} \geq 1, i=1, \ldots, n$. By $\left(\widetilde{\mathrm{H}}_{2}\right)$ and the above inequality, it follows that

$$
\begin{aligned}
f_{i}\left(t, x^{\sigma}(t)\right) & \geq\left(\frac{1}{K}\right)^{\lambda_{i i}} f_{i}\left(t, x_{1}^{\sigma}(t), \ldots, \frac{K x_{i}^{\sigma}(t)}{e^{\sigma}(t)} e^{\sigma}(t), \ldots, x_{n}^{\sigma}(t)\right) \\
& \geq K^{\mu_{i i}-\lambda_{i i}}\left(\frac{x_{i}^{\sigma}(t)}{e^{\sigma}(t)}\right)^{\mu_{i i}} f_{i}\left(t, x_{1}^{\sigma}(t), \ldots, e^{\sigma}(t), \ldots, x_{n}^{\sigma}(t)\right) \\
& \geq K^{\mu_{i j}-\lambda_{i i}} I_{i 1}^{\mu_{i i}} f_{i}\left(t, x_{1}^{\sigma}(t), \ldots, e^{\sigma}(t), \ldots, x_{n}^{\sigma}(t)\right) \\
& \geq K^{\sum_{j=1}^{n}\left(\mu_{i j}-\lambda_{i j}\right)} I_{i 1}^{\mu_{i i}} \prod_{j=1, j \neq i}^{n} I_{j 2}^{\mu_{i j}} f_{i}\left(t, E^{\sigma}(t)\right),
\end{aligned}
$$

hence

$$
\begin{aligned}
& \int_{a}^{\sigma(b)} f_{i}\left(s, E^{\sigma}(s)\right) \Delta s \\
& \quad \leq K^{\sum_{j=1}^{n}\left(\lambda_{i j}-\mu_{i j}\right)} I_{i 1}^{-\mu_{i i}} \prod_{j=1, j \neq i}^{n} I_{j 2}^{-\mu_{i j}} \int_{a}^{\sigma(b)} f_{i}\left(s, x^{\sigma}(s)\right) \Delta s \\
& \quad=K^{\sum_{j=1}^{n}\left(\lambda_{i j}-\mu_{i j}\right)} I_{i 1}^{-\mu_{i i}} \prod_{j=1, j \neq i}^{n} I_{j 2}^{-\mu_{i j}}\left(-x_{i}^{\Delta}(\sigma(b))+x_{i}^{\Delta}(a)\right)<\infty .
\end{aligned}
$$

Sufficiency. Suppose that there exists a constant $C \geq 1$ such that $C I_{i 1} \geq 1$ and $I_{i 2} \leq C$.

We consider

$$
\begin{aligned}
& \alpha_{i}(t)=k_{i 1} y_{i}(t), \\
& \beta_{i}(t)=k_{i 2} y_{i}(t),
\end{aligned}
$$

with

$$
y_{i}(t)=\int_{a}^{\sigma(b)} G(t, s) f_{i}\left(s, E^{\sigma}(s)\right) \Delta s,
$$

where $G(t, s)$ is Green's function (2.2) and $k_{i 1}$ and $k_{i 2}$ are determined below. 
Note that $y_{i}$ satisfies

- $y_{i}, y_{i}^{\Delta} \in C\left(\left[a, \sigma^{2}(b)\right]_{\mathbb{T}}\right)$,

- $y_{i}^{\Delta \Delta} \in C_{r d}\left(\left(a, \sigma^{2}(b)\right)_{\mathbb{T}}\right)$.

We have

$$
e(t) I_{i 1} \leq y_{i}(t) \leq e(t) I_{i 2},
$$

where

$$
I_{i 1}=\frac{1}{\sigma^{2}(b)-a} \int_{a}^{\sigma(b)} \frac{(s-a)\left(\sigma^{2}(b)-\sigma(s)\right)}{\sigma^{2}(b)-a} f_{i}\left(s, e^{\sigma}(s)\right) \Delta s,
$$

and

$$
I_{i 2}=\int_{a}^{\sigma(b)} f_{i}\left(s, e^{\sigma}(s)\right) \Delta s
$$

Consider now

$$
\begin{aligned}
& k_{i 1}=\min \left\{1,\left(C^{\sum_{j=1}^{n}\left(\lambda_{i j}-\mu_{i j}\right)} \prod_{j=1}^{n} I_{j 2}^{\lambda_{i j}}\right)^{\frac{1}{1-\mu_{i i}}}\right\}, \\
& k_{i 2}=\max \left\{1,\left(C^{\sum_{j=1}^{n}\left(\mu_{i j}-\lambda_{i j}\right)} \prod_{j=1}^{n} I_{j 1}^{\lambda_{i j}}\right)^{\frac{1}{1-\mu_{i i}}}\right\} .
\end{aligned}
$$

Given that $e^{\sigma}(t) I_{i 1} \leq y_{i}^{\sigma}(t)$ and $C I_{i 1} \geq 1$, then $e^{\sigma}(t) \leq C y_{i}^{\sigma}(t)$. Since

$$
f_{i}\left(t, \alpha_{1}^{\sigma}(t), \ldots, \alpha_{n}^{\sigma}(t)\right)=f_{i}\left(t, \frac{k_{11}}{C} \frac{C y_{1}^{\sigma}(t)}{e^{\sigma}(t)} e^{\sigma}(t), \ldots, \frac{k_{n 1}}{C} \frac{C y_{n}^{\sigma}(t)}{e^{\sigma}(t)} e^{\sigma}(t)\right)
$$

we have that

$$
\begin{aligned}
f_{i}\left(t, \alpha^{\sigma}(t)\right) & \geq \prod_{j=1}^{n}\left(\frac{k_{j 1}}{C}\right)^{\mu_{i j}}\left(\frac{C y_{j}^{\sigma}(t)}{e^{\sigma}(t)}\right)^{\lambda_{i j}} f_{i}\left(t, E^{\sigma}(t)\right) \\
& \geq \prod_{j=1}^{n} k_{j 1}^{\mu_{i j}} C^{\lambda_{i j}-\mu i j} I_{j 2}^{\lambda_{i j}} f_{i}\left(t, E^{\sigma}(t)\right),
\end{aligned}
$$

and

$$
\prod_{j=1}^{n} k_{j 1}^{\mu_{i j}} C^{\lambda_{i j}-\mu i j} I_{j 2}^{\lambda_{i j}} \geq k_{i 1}^{1-\mu_{i i}} \prod_{j=1}^{n} k_{j 1}^{\mu_{i j}} \geq k_{i 1} \prod_{j=1, i \neq j}^{n} k_{j 1}^{\mu_{i j}} \geq k_{i 1},
$$

which implies that

$$
f_{i}\left(t, \alpha^{\sigma}(t)\right) \geq k_{i 1} f_{i}\left(t, E^{\sigma}(t)\right)=-\alpha_{i}^{\Delta \Delta}(t) .
$$


In a similar way,

$$
\begin{aligned}
f_{i}\left(t, \beta^{\sigma}(t)\right) & \leq \prod_{j=1}^{n}\left(k_{j 2} C\right)^{\mu_{i j}}\left(\frac{y_{j}^{\sigma}(t)}{C e^{\sigma}(t)}\right)^{\lambda_{i j}} f_{i}\left(t, E^{\sigma}(t)\right) \\
& \leq \prod_{j=1}^{n} k_{j 2}^{\mu_{i j}} C^{\mu_{i j}-\lambda i j} I_{j 1}^{\lambda_{i j}} f_{i}\left(t, E^{\sigma}(t)\right) \leq k_{i 2} f_{i}\left(t, E^{\sigma}(t)\right)=-\beta_{i}^{\Delta \Delta}(t) .
\end{aligned}
$$

Thus, there is a lower solution $\alpha$ and an upper solution $\beta$ of problem $\left(P_{0}\right)$ that satisfy $0<\alpha_{i}(t) \leq \beta_{i}(t)$ for $t \in\left(J^{\kappa}\right)^{o}, i=1, \ldots, n, \alpha_{i}(a)=\beta_{i}(a)=\alpha_{i}\left(\sigma^{2}(b)\right)=\beta_{i}\left(\sigma^{2}(b)\right)=0$. Applying Lemma 3.2, problem $\left(P_{0}\right)$ has a solution $x$ such that $\alpha \leq x \leq \beta$. Note that for $t \in\left(J^{\kappa}\right)^{o}$ and $\alpha \leq x \leq \beta$,

$$
\begin{aligned}
0 & \leq f_{i}\left(t, x_{i}^{\sigma}(t)\right)=f_{i}\left(t,\left(\frac{k_{11}}{C}\right)\left(\frac{C x_{1}^{\sigma}(t)}{k_{11} e^{\sigma}(t)}\right) e^{\sigma}(t), \ldots,\left(\frac{k_{n 1}}{C}\right)\left(\frac{C x_{n}^{\sigma}(t)}{k_{n 1} e^{\sigma}(t)}\right) e^{\sigma}(t)\right) \\
& \leq\left(\frac{k_{11}}{C}\right)^{\lambda_{i 1}}\left(\frac{C x_{1}^{\sigma}(t)}{k_{11} e^{\sigma}(t)}\right)^{\mu_{i 1}} \ldots\left(\frac{k_{n 1}}{C}\right)^{\lambda_{i n}}\left(\frac{C x_{n}^{\sigma}(t)}{k_{n 1} e^{\sigma}(t)}\right)^{\mu_{i n}} f_{i}\left(t, E^{\sigma}(t)\right) \leq h_{i}(t),
\end{aligned}
$$

with $h_{i}(t)=K_{i} f_{i}\left(t, E^{\sigma}(t)\right)$ and

$$
K_{i}=\prod_{j=1}^{n}\left(\frac{k_{j 1}}{C}\right)^{\lambda_{i j}-\mu_{i j}} \prod_{j=1}^{n} \max _{k=1, \ldots, n}\left\{\left(I_{k 1}\right)^{\mu_{i j}},\left(I_{k 2}\right)^{\mu_{i j}}\right\} .
$$

Due to the hypothesis, we can then ensure that

$$
\int_{a}^{\sigma(b)} h_{i}(s) \Delta s<\infty
$$

for $i=1, \ldots, n$, which implies the existence of a positive solution of type 1 of problem $\left(P_{0}\right)$ such that $0<\alpha \leq x \leq \beta$.

Theorem 3.2 If there exists a positive solution of the problem and $\sigma^{2}(b)>0$, then the following conditions hold:

$$
0<\int_{a}^{\sigma(b)}(\sigma(s)-a)(\sigma(b)-\sigma(s)) f_{i}\left(s,\left[\sigma^{2}(b)\right]\right) \Delta s<\infty
$$

for all $i=1, \ldots, n$, with $\left[\sigma^{2}(b)\right]=\left(\sigma^{2}(b), \ldots, \sigma^{2}(b)\right)$.

Proof Fix $i=1, \ldots, n$, let us consider $C_{0}>0, C_{0}^{i}$ to be two constants such that $\frac{C_{0} x_{j}^{\sigma}(t)}{\sigma^{2}(b)} \leq 1$ if $j=1, \ldots, n$ and $j \neq i, \frac{1}{C_{0}} \geq 1, \frac{C_{0}^{i} x_{i}^{\sigma}(t)}{\sigma^{2}(b)} \geq 1$ and $C_{0}^{i} \geq 1$.

We have

$$
\begin{aligned}
f_{i}\left(t, x^{\sigma}(t)\right) & \geq\left(\prod_{j=1, j \neq i}^{n}\left(\frac{1}{C_{0}}\right)^{\lambda i j}\left(\frac{C_{0} x_{j}^{\sigma}(t)}{\sigma^{2}(b)}\right)^{\mu i j}\right) f_{i}\left(t, \sigma^{2}(b), \ldots, x_{i}^{\sigma}(t), \ldots, \sigma^{2}(b)\right) \\
& \geq\left(\prod_{j=1, j \neq i}^{n}\left(\frac{1}{C_{0}}\right)^{\lambda i j}\left(\frac{C_{0} x_{j}^{\sigma}(t)}{\sigma^{2}(b)}\right)^{\mu i j}\right)\left(\frac{1}{C_{0}^{i}}\right)^{\mu i i}\left(\frac{C_{0}^{i} x_{i}^{\sigma}(t)}{\sigma^{2}(b)}\right)^{\lambda i i} f_{i}\left(t,\left[\sigma^{2}(b)\right]\right) .
\end{aligned}
$$


Hence,

$$
f_{i}\left(t,\left[\sigma^{2}(b)\right]\right) \leq M f_{i}\left(t, x^{\sigma}(t)\right)
$$

where

$$
M=\sup _{t \in[a, \sigma(b)]_{\mathbb{T}}}\left(\prod_{j=1, j \neq i}^{n} C_{0}^{\lambda i j-\mu i j}\left(\frac{x_{j}^{\sigma}(t)}{\sigma^{2}(b)}\right)^{-\mu i j}\right)\left(C_{0}^{i}\right)^{\mu i i}\left(\frac{C_{0}^{i} x_{i}^{\sigma}(t)}{\sigma^{2}(b)}\right)^{-\lambda i i} .
$$

Let $t_{i} \in(a, \sigma(b))_{\mathbb{T}}$,

$$
\begin{aligned}
& \int_{a}^{t_{i}} \int_{t}^{t_{i}} f_{i}\left(s,\left[\sigma^{2}(b)\right]\right) \Delta s \Delta t \\
& \quad \leq-M \int_{a}^{t_{i}} \int_{t}^{t_{i}} x_{i}^{\Delta \Delta}(s) \Delta s \Delta t \\
& \quad=-M \int_{a}^{t_{i}}\left(x_{i}^{\Delta}\left(t_{i}\right)-x_{i}^{\Delta}(t)\right) \Delta t=-M\left(x_{i}^{\Delta}\left(t_{i}\right)\left(t_{i}-a\right)-x_{i}\left(t_{i}\right)+x_{i}(a)\right)<\infty .
\end{aligned}
$$

Integrating by parts, we have

$$
\int_{a}^{t_{i}} \int_{t}^{t_{i}} f_{i}\left(s,\left[\sigma^{2}(b)\right]\right) \Delta s \Delta t=\int_{a}^{t_{i}}(\sigma(t)-a) f_{i}\left(t, x_{\sigma^{2}(b)}\right) \Delta t<\infty .
$$

In a similar way,

$$
\begin{aligned}
\int_{t_{i}}^{\sigma(b)} \int_{t_{i}}^{t} f_{i}\left(s,\left[\sigma^{2}(b)\right]\right) \Delta s \Delta t & \leq-M \int_{t_{i}}^{\sigma(b)} \int_{t_{i}}^{t} x_{i}^{\Delta \Delta}(s) \Delta s \Delta t \\
& =-M \int_{t_{i}}^{\sigma(b)}\left(x_{i}^{\Delta}(t)-x_{i}^{\Delta}\left(t_{i}\right)\right) \Delta t \\
& =-M\left(x_{i}(\sigma(b))-x_{i}\left(t_{i}\right)-x_{i}^{\Delta}\left(t_{i}\right)\left(\sigma(b)-t_{i}\right)\right)<\infty
\end{aligned}
$$

and integrating by parts,

$$
\int_{t_{i}}^{\sigma(b)} \int_{t_{i}}^{t} f_{i}\left(s,\left[\sigma^{2}(b)\right]\right) \Delta s \Delta t=\int_{t_{i}}^{\sigma(b)}(\sigma(b)-\sigma(t)) f_{i}\left(t,\left[\sigma^{2}(b)\right]\right) \Delta t<\infty .
$$

Then we conclude that

$$
0<\int_{a}^{\sigma(b)}(\sigma(t)-a)(\sigma(b)-\sigma(t)) f_{i}\left(t,\left[\sigma^{2}(b)\right]\right) \Delta t<\infty
$$

Theorem 3.3 If the following conditions hold:

$$
0<\int_{a}^{\sigma(b)}(\sigma(s)-a)(\sigma(b)-\sigma(s)) f_{i}\left(s,\left[\sigma^{2}(b)\right]\right) \Delta s<\infty
$$

for all $i=1, \ldots, n$ and $\sigma^{2}(b)>0$, then there exists a lower solution to problem $(P)$. 
Proof Consider the function

$$
g_{i}(t)=\int_{a}^{\sigma(b)} G(t, s) \frac{(\sigma(s)-a)\left(\sigma^{2}(b)-\sigma(s)\right)}{\left(\sigma^{2}(b)-a\right)^{2}} f_{i}\left(s,\left[\sigma^{2}(b)\right]\right) \Delta s .
$$

Let us see that

$$
\begin{aligned}
& 0 \leq g_{i}(t)<\infty \\
& \int_{a}^{\sigma(b)} G(t, s)\left(\frac{(\sigma(s)-a)\left(\sigma^{2}(b)-\sigma(s)\right)}{\left(\sigma^{2}(b)-a\right)^{2}}\right)^{\mu_{i i}} f_{i}\left(s,\left[\sigma^{2}(b)\right]\right) \Delta s \\
& \quad \int_{a}^{t} \frac{(\sigma(s)-a)\left(\sigma^{2}(b)-t\right)}{\sigma^{2}(b)-a}\left(\frac{(\sigma(s)-a)\left(\sigma^{2}(b)-\sigma(s)\right)}{\left(\sigma^{2}(b)-a\right)^{2}}\right)^{\mu_{i i}} f_{i}\left(s,\left[\sigma^{2}(b)\right]\right) \Delta s \\
& \quad+\int_{t}^{\sigma(b)} \frac{(t-a)\left(\sigma^{2}(b)-\sigma(s)\right)}{\sigma^{2}(b)-a}\left(\frac{(\sigma(s)-a)\left(\sigma^{2}(b)-\sigma(s)\right)}{\left(\sigma^{2}(b)-a\right)^{2}}\right)^{\mu_{i i}} f_{i}\left(s,\left[\sigma^{2}(b)\right]\right) \Delta s \\
& \leq \int_{a}^{\sigma(b)} \frac{(\sigma(s)-a)\left(\sigma^{2}(b)-\sigma(s)\right)}{\sigma^{2}(b)-a}\left(\frac{(\sigma(s)-a)\left(\sigma^{2}(b)-\sigma(s)\right)}{\left(\sigma^{2}(b)-a\right)^{2}}\right)^{\mu_{i i}} f_{i}\left(s,\left[\sigma^{2}(b)\right]\right) \Delta \\
& \leq \frac{1}{\sigma^{2}(b)-a} \int_{a}^{\sigma(b)}(\sigma(s)-a)\left(\sigma^{2}(b)-\sigma(s)\right) f_{i}\left(s,\left[\sigma^{2}(b)\right]\right) \Delta s<\infty .
\end{aligned}
$$

Furthermore, $g_{i}(a)=g_{i}\left(\sigma^{2}(b)\right)=0$.

If we consider

$$
L_{i 1}=\frac{1}{\sigma^{2}(b)-a} \int_{a}^{\sigma(b)}\left(\frac{(\sigma(s)-a)\left(\sigma^{2}(b)-\sigma(s)\right)^{1+\mu_{i i}}}{\left(\sigma^{2}(b)-a\right)^{2 \mu_{i i}}}\right) f_{i}\left(s,\left[\sigma^{2}(b)\right]\right) \Delta s .
$$

Then

$$
g_{i}(t) \leq L_{i 1}
$$

On the other hand,

$$
\begin{aligned}
& \frac{(t-a)\left(\sigma^{2}(b)-t\right)}{\left(\sigma^{2}(b)-a\right)^{2}} L_{i 1} \\
& \leq \int_{a}^{t} \frac{(\sigma(s)-a)\left(\sigma^{2}(b)-t\right)}{\sigma^{2}(b)-a}\left(\frac{(\sigma(s)-a)\left(\sigma^{2}(b)-\sigma(s)\right)}{\left(\sigma^{2}(b)-a\right)^{2}}\right)^{\mu_{i i}} f_{i}\left(s,\left[\sigma^{2}(b)\right]\right) \Delta s \\
& \quad+\int_{t}^{\sigma(b)} \frac{(t-a)\left(\sigma^{2}(b)-\sigma(s)\right)}{\sigma^{2}(b)-a}\left(\frac{(\sigma(s)-a)\left(\sigma^{2}(b)-\sigma(s)\right)}{\left(\sigma^{2}(b)-a\right)^{2}}\right)^{\mu_{i i}} f_{i}\left(s,\left[\sigma^{2}(b)\right]\right) \Delta s \\
& =g_{i}(t) .
\end{aligned}
$$

Let $\alpha_{i}(t)=k_{i 1} g_{i}(t)$, where

$$
k_{i 1}=\min \left\{1, \prod_{j=1}^{n} L_{j 1}^{\mu_{i j}}\left(\frac{1}{\sigma^{2}(b)}\right)^{\lambda_{i j}} C_{2}^{\mu i j-\lambda i j}\right\}^{\frac{1}{1-\mu_{i i}}}
$$

with $C_{2}$ being a constant such that $\sigma^{2}(b) C_{2} L_{i 1} \leq 1$ and $\frac{1}{C_{2} \sigma^{2}(b)} \geq 1$. 
Thus, if we note that $0<\mu_{i i}<1$ and $\mu_{i j}<0$ if $i \neq j$, we obtain

$$
k_{i 1} L_{i 1}\left[\frac{(t-a)\left(\sigma^{2}(b)-t\right)}{\left(\sigma^{2}(b)-a\right)^{2}}\right]^{\mu i i} \leq \alpha_{i}(t)^{\mu_{i i}},
$$

and

$$
\alpha_{j}(t)^{\mu_{i j}} \geq L_{j 1}^{\mu_{i j}} .
$$

Hence,

$$
\begin{aligned}
f_{i}\left(t, \alpha^{\sigma}(t)\right) & \geq \prod_{j=1}^{n}\left(\frac{1}{C_{2} \sigma^{2}(b)}\right)^{\lambda_{i j}}\left(C_{2} \alpha_{i}^{\sigma}(t)\right)^{\mu_{i j}} f_{i}\left(t,\left[\sigma^{2}(b)\right]\right) \\
& =\prod_{j=1}^{n} \frac{1}{\sigma^{2}(b)^{\lambda_{i j}}} C_{2}^{\mu_{i j}-\lambda_{i j}}\left(\alpha_{j}^{\sigma}(t)\right)^{\mu_{i j}} f_{i}\left(t,\left[\sigma^{2}(b)\right]\right) \\
& \geq \prod_{j=1, j \neq i}^{n} \frac{1}{\sigma^{2}(b)^{\lambda_{i i}+\lambda_{i j}}} C_{2}^{\mu_{i j}-\lambda_{i j}} C_{2}^{\mu_{i i}-\lambda_{i i}} L_{j 1}^{\mu_{i j}}\left(\alpha_{i}^{\sigma}(t)\right)^{\mu_{i i}} f_{i}\left(t,\left[\sigma^{2}(b)\right]\right) \\
& \geq k_{i 1}\left(\frac{\left(\sigma^{2}(b)-\sigma(t)\right)(\sigma(t)-a)}{\left(\sigma^{2}(b)-a\right)^{2}}\right)^{\mu_{i i}} f_{i}\left(t,\left[\sigma^{2}(b)\right]\right)=-\alpha_{i}^{\Delta \Delta}(t) .
\end{aligned}
$$

This implies that $\alpha$ is a lower solution of problem $\left(P_{0}\right)$.

Theorem 3.4 Suppose that the conditions of the above theorem are satisfied and consider $\alpha$ the lower solution of problem $\left(P_{0}\right)$ provided. If there exists $\beta$, an upper solution of $\left(P_{0}\right)$, with $0<\alpha \leq \beta$ and $\left(\widetilde{\mathrm{H}}_{1}\right)$ and $\left(\mathrm{H}_{3}\right)$ or $\left(\overline{\mathrm{H}}_{3}\right)$ hold, then there exists $x$ a positive solution of $\left(P_{0}\right)$.

Proof The demonstration of this fact is immediate taking into account the construction of the lower solution $\alpha$ obtained in the previous theorem, the existence of the upper solution $\beta$ with $0<\alpha \leq \beta$ and the implementation of Lemma 3.2.

\subsection{Particular cases}

Let us briefly consider the following examples.

1 If $\left[a, \sigma^{2}(b)\right]_{\mathbb{T}}$ is bounded and consists of only isolated points such as in the case $\mathbb{T}=h \mathbb{Z}$, then the conditions of Theorems 3.1 and 3.2 are fulfilled. This follows from the fact

$$
\int_{a}^{\sigma^{2}(b)} f(t) \Delta t=\sum_{t \in\left[a, \sigma^{2}(b)\right)}(\sigma(t)-t) f(t) .
$$

2 Let $q>1$ be fixed, the quantum time scale $\overline{q^{\mathbb{Z}}}$ is defined as

$$
\overline{q^{\mathbb{Z}}}=\left\{q^{k}: k \in \mathbb{Z}\right\} \cup\{0\},
$$

which appears throughout the mathematical physics literature, where the dynamical systems of interest are the q-difference equations. 
Since the only non-isolated point is 0 , the interesting case is the one in which the interval contains this point. We consider $a=0$ and $\sigma(b)=1$.

Taking into account the fact that

$$
\int_{0}^{1} f(t) \Delta t=\sum_{k=1}^{\infty} \frac{q-1}{q^{k}} f\left(q^{-k}\right)
$$

we have

$$
\int_{0}^{1} f_{i}\left(s, E^{\sigma}(s)\right) \Delta s=\sum_{k=1}^{\infty} \frac{q-1}{q^{k}} f_{i}\left(q^{-k}, E^{\sigma}\left(q^{-k}\right)\right)
$$

with $E^{\sigma}\left(q^{-k}\right)=\left(q^{-k+1}\left(1-q^{-k}\right), \ldots, q^{-k+1}\left(1-q^{-k}\right)\right)$. Hence, the convergence of this series is the necessary and sufficient condition in Theorem 3.1.

Analogously, the condition in Theorem 3.2 can be rewritten as

$$
0<\int_{0}^{1} q^{-2 k+1}(q-1)\left(1-q^{-k+1}\right) f_{i}\left(q^{-k},(q, \ldots, q)\right) \Delta s<\infty .
$$

\section{Competing interests}

The authors declare that they have no competing interests.

\section{Authors' contributions}

All authors contributed equally in this article. They read and approved the final manuscript.

\section{Acknowledgements}

The authors are grateful to the referees for their valuable suggestions that led to the improvement of the original manuscript.The research of VOE has been partially supported by Ministerio de Educación y Ciencia (Spain) and FEDER, Project MTM2010-15314. The research of AL has been supported by FSE y de la DX de Ordenación y Calidad del Sistema Universitario de Galicia, de la Conselleria de Educación y Ordenación Universitaria-Xunta de Galicia.

Received: 6 June 2012 Accepted: 15 October 2012 Published: 29 October 2012

\section{References}

1. Hilger, S: Ein Maßkettenkalkül mit Anwendung auf Zentrumsmannigfaltigkeiten. Universität Würzburg (1988) (in German)

2. Jain, B, Sheng, AD: An exploration of the approximation of derivative functions via finite differences. Rose-Hulman Undergraduate Math J. 8, 1-19 (2007)

3. Atici, FM, Biles, DC, Lebedinsky, A: An application of time scales to economics. Math. Comput. Model. 43, 718-726 (2006)

4. Wong, JSW: On the generalized Emden-Fowler equation. SIAM Rev. 17, 339-360 (1975)

5. Agarwal, RP, O'Regan, D, Lakshmikantham, V, Leela, S: An upper and lower solution theory for singular Emden-Fowler equations. Nonlinear Anal., Real World Appl. 3, 275-291 (2002)

6. Habets, P, Zanolin, F: Upper and lower solutions for a generalized Emden-Fowler equations. J. Math. Anal. Appl. 181, 684-700 (1994)

7. Zhang, Y: Positive solutions of singular sublinear Emden-Fowler boundary value problems. J. Math. Anal. Appl. 185, 215-222 (1994)

8. Wei, Z: Positive solution of singular Dirichlet boundary value problems for second order differential equation system. J. Math. Anal. Appl. 328, 1255-1267 (2007)

9. Agarwal, RP, Bohner, M, Grace, SR, O'Regan, D: Discrete Oscillation Theory. Hindawi Publishing Corporation, New York (2005)

10. Akin-Bohner, E, Hoffacker, J: Oscillation properties of an Emden-Fowler type equation on discrete time scales. J. Differ. Equ. Appl. 9, 603-612 (2003)

11. Gómez, A, Otero-Espinar, V: Existence and uniqueness of positive solution for singular BVPs on time scales. Adv. Differ. Equ. 2009, Art. ID 728484 (2009)

12. Khan, RA, Nieto, JJ, Otero-Espinar, V: Existence and approximation of solution of three-point boundary value problems on time scale. J. Differ. Equ. Appl. 14, 723-736 (2008)

13. Zhao, J, Lian, $\mathrm{H}, \mathrm{Ge}, \mathrm{W}$ : Existence of positive solutions for nonlinear m-point boundary value problems on time scales. Bound. Value Probl. 2012, 4 (2012) 
14. Agarwal, RP, Otero-Espinar, V, Perera, K, Vivero, DR: Multiple positive solutions in the sense of distributions of singular BVPs on time scales and a application to Emden-Fowler equations. Adv. Differ. Equ. 2008, Article ID 796851 (2008)

15. Han, Z, Sun, S, Shi, B: Oscillation criteria for a class of second-order Emden-Fowler delay dynamic equations on time scales. J. Math. Anal. Appl. 334, 847-858 (2007)

16. Bohner, M, Erbe, L, Peterson, A: Oscillation for nonlinear second order dynamic equations on a time scale. J. Math. Anal. Appl. 301, 491-507 (2005)

17. Bohner, M, Peterson, A: Dynamic Equations on Time Scales: An Introduction with Applications. Birkhauser, Boston (2001)

18. Hartman, P: Ordinary Differential Equations. Birkhauser, Boston (1982)

doi:10.1186/1687-1847-2012-185

Cite this article as: Lago et al.: Positive solution of singular BVPs for a system of dynamic equations on time scales. Advances in Difference Equations 2012 2012:185.

Submit your manuscript to a SpringerOpen ${ }^{\odot}$ journal and benefit from:

- Convenient online submission

- Rigorous peer review

- Immediate publication on acceptance

- Open access: articles freely available online

- High visibility within the field

- Retaining the copyright to your article 